\title{
Control Theory and Deep Brain Stimulation for Relief from Neurological Diseases
}

\author{
Clare M. Davidson, Annraoi M. dePaor, Madeleine M. Lowery*
}

ISchool of Electrical, Electronic and Communications Engineering, National University of Ireland, Dublin (UCD), Belfield, Dublin 4, Ireland.

\section{BIOGRAPHICAL NOTES}

Clare Davidson, is a PhD student in the School of Electrical, Electronic and Communications Engineering, University College Dublin. Her research focuses on the theoretical and computational modeling of the basal ganglia in the brain, and the application of deep brain stimulation for the treatment of Parkinson's disease. She received the B.Sc (Physiology) and the B.E. (Electronic) from University College Dublin in 2004 and 2010 respectively. She is a student member of the IEEE and the Society for Neuroscience.

Professor Annraoi de Paor, was born in Waterford, Ireland in 1940. He received the degrees of BE (1961), PhD (1967) and DSc (1974) from the National University of Ireland (NUI), Dublin (which also trades under its older name of University College Dublin (UCD)), and the degree of MS (1963) from the University of California at Berkeley. He was a lecturer in Electrical Engineering at the University of Salford, UK, from 1963 to 1967, and at NUI Dublin from 1967 to 1969. In 1969 he was appointed Professor of Control Engineering at the University of Salford and on 1 January, 1978 became Professor of Electrical Engineering at NUI Dublin. He has been Professor Emeritus since 2005, but remains active in teaching and research. Over the years, he has taught, supervised research students and conducted research in Control Theory, Biomedical Engineering, Rehabilitation Engineering, Renewable Energy and Engineering Education. In 1986 he established a Research Laboratory at Ireland's National Rehabilitation Hospital, devoted to improving the quality of life for seriously disabled people through the development of Assistive Technology, and directed that laboratory until his retirement. In 1996, he was co-founder of the interdisciplinary Centre for Disability Studies at NUI Dublin and directed its taught postgraduate courses for the next five years. He continues to teach and supervise students in that Centre. He is a Member of the Royal Irish Academy and a Fellow of the Institution of Engineers of Ireland, the Institution of Engineering and Technology (formerly IEE) and the Institute of Mathematics and its Applications. He is a Member of the Institute of Measurement and Control. He directs an annual Summer School, devoted to all aspects of the Gaelic culture of Ireland, in the Irish-speaking area (Gaeltacht) of his native county.

Dr Madeleine Lowery, is a Senior Lecturer in the School of Electrical, Electronic and Communications Engineering, University College Dublin. Her research involves the exploration of nerve and muscle activity through mathematical modeling, analysis, and experimentation, to increase understanding of neuromuscular activity in healthy and diseased states and develop more effective rehabilitation strategies and therapies. Her research interests include electromyography, myoelectric control of artificial limbs, bioelectromagnetics, deep brain stimulation and neural control of movement. Dr Lowery received the B.E. and Ph.D. degrees from the Department of Electronic and Electrical Engineering, University College Dublin, in 1996 and 2000, respectively. 
Between 2000 and 2005, she was a Postdoctoral Fellow then Research Assistant Professor at the Rehabilitation Institute of Chicago and the Department of Physical Medicine and Rehabilitation, Northwestern University. She is a member of the IEEE and a member of the Council of the International Society of Electrophysiology and Kinesiology (ISEK).

\section{KEY WORDS}

Deep Brain Stimulation, Parkinson's disease, Essential Tremor, Control Theory.

\section{ABSTRACT}

Deep Brain Stimulation (DBS) is a neurosurgical technique in which microelectrodes, embedded in a closely specified target area of the brain, are stimulated by a periodic, rectangular, charge-balanced signal, generated by a stimulator embedded in the chest. It was serendipitously discovered by neurosurgeon Professor Alim-Louis Benabid in Grenoble in 1987 that "high frequency" (of the order of $130 \mathrm{~Hz}$ ) stimulation of the Basal Ganglia in the brain could eliminate the characteristic tremor (about 5 to $8 \mathrm{~Hz}$ ) associated with Parkinson's disease and also release the associated "locking of gait." Since then the idea has been applied to other neurological diseases such as epilepsy, depression and Tourette syndrome and the phenomenon of "essential tremor."The mode of action of DBS is still incompletely understood. This paper presents attempts made by the authors to develop constructive models of the phenomenology of DBS, based on classical Control Theory tools such as the Nyquist Stability Criterion, the Describing Function, the Root Locus method, Liapunov's theorem of the First Approximation and, above all, the concept of the Equivalent Nonlinearity associated with injection of a "high frequency" wave into a nonlinear feedback loop to quench troublesome "low frequency" oscillations. These models have proved very successful in predicting the dependence of pathological oscillations, observed as Local Field Potentials (LFP), on amplitude and fractional pulse duration of the DBS signal.

\section{Introduction}

Several neurological disorders - some progressive, others not - have been linked to pathological electrical oscillations in various areas of the brain.
These include Parkinson's disease, epilepsy, dystonias, cluster headaches, obsessive-compulsive disorder, Gilles de la Tourette tics and depression [1]. Relief from the symptoms of such conditions has been strongly correlated [2] with quenching of these oscillations by deep brain stimulation (DBS), although a strictly causal relation between oscillations and symptoms does not appear to have been established yet. Nonetheless, quenching of the oscillations accompanies relief from the symptoms with such high probability that it has inspired our work in this area. We shall concentrate on two conditions here: Parkinson's disease [3] and essential tremor [4], the former progressive, the latter not.

Parkinson's disease is correlated with pathological oscillations in the basal ganglia of the brain, one on each side, shown schematically in Fig. 1. It was discovered by Arvid Carlsson in the 1950s that a chemical transmitter substance called dopamine is synthesized in the substantia nigra pars compacta (SNc) and that it is the selective death of these cells which manifests itself as Parkinson's disease. Carlsson was awarded the Nobel Prize for this discovery in 2000 [5]. It was discovered serendipitously by Benabid in 1987 [6] that DBS of the basal ganglia - most commonly targeting the subthalamic nucleus (STN) - can, with careful choice of frequency, $1 / T$, amplitude, $\alpha$, and fractional pulsewidth, $\alpha$ of the charge balanced DBS waveform shown on Fig. 2, give almost complete relief from the symptoms of Parkinson's disease. A DBS frequency $1 / T=130 \mathrm{~Hz}$ is typical in clinical practice, and we shall use it below. The pathological oscillations whose extinction is brought about by DBS are mainly observed in the tremor band (about 4 $8 \mathrm{~Hz}$ ), the beta band (about $12-30 \mathrm{~Hz}$ ) and the gamma band (> $30 \mathrm{~Hz})$. The tremor band oscillations appear to underlie the observed tremor, while the beta band oscillations are implicated in the seizure of gait and difficulty in initiating planned actions [2]. The gamma band oscillations are generally considered benign, although de Paor and Lowery have demonstrated that bilateral coupling of feedback loops oscillating in the tremor band and the gamma band can give rise to beta band oscillations [7]

We have found that the complicated intercoupling of feedback loops in the basal ganglia (one of which, STN-GPe, has been studied in vitro [8]) is not necessary to model quite accurately the ob- 
served phenomenology of quenching the pathological oscillations. We have succeeded in bringing the following facets of control theory successfully to bear on the problem: Liapunov's theorem of the first approximation, the Root Locus method, the Describing Function, the Nyquist Criterion and the concept of the Equivalent Nonlinearity. These are all part of the stock-in-trade of engineers from many branches and all but the last are covered in very many undergraduate textbooks. The basic principles of the Equivalent Nonlinearity are dealt with in only two textbooks with which we are familiar $[9,10]$. It was developed from a fairly primitive base into a powerful problem-solving tool by Simpson and Power [11].

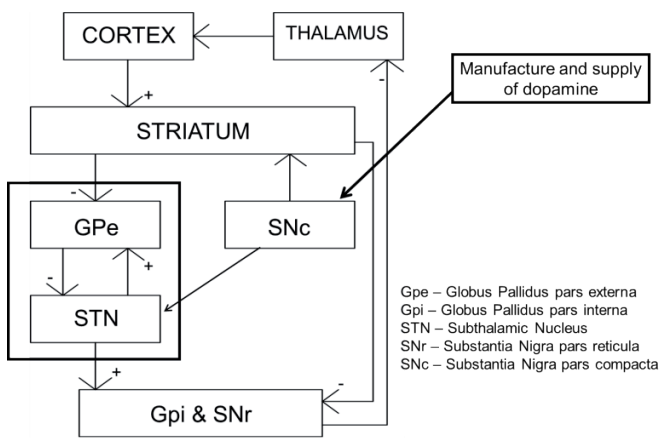

Fig. 1: Schematic of the basal ganglia.

In our studies of pathological oscillations in the basal ganglia we have followed the practice of characterizing them by the local field potential (LFP) which is the potential difference between an electrode placed in the close neighborhood of the oscillating cells and a reference electrode. We have been strongly influenced by the works of Rosenblum and Pikovsky [12] and David and Friston [13]. The former have shown in simulated interconnections of several thousand model neurons that, when a certain coupling coefficient exceeds a threshold value, pathological oscillations ensue. These evolve in accordance with second order dynamics, which they analyze using ideas expounded by Kuramoto [14]. Further details and applications of some of the concepts invoked here are given in [15].

Control theory deals - inter alia - with the dynamics of oscillations in nonlinear feedback loops. This suggested to us the basic structure shown in Fig. 2. The sigmoid nonlinearity is very widely used in biological feedback loops $[13,16]$. Symmetric and asymmetric sigmoids are found in the literature. We have chosen a symmetrical sigmoid here, which, in conjunction with the DBS waveform shown in Fig. 2, ensures that the Describing Functions involved are real and positive.

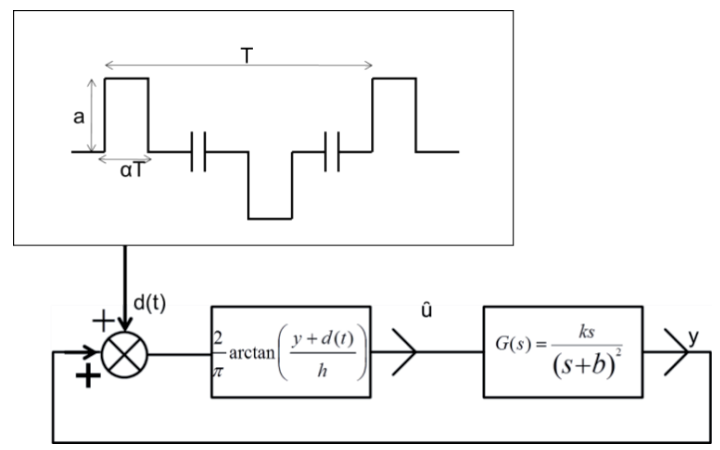

Fig. 2: The feedback loop and DBS waveform used to study Parkinsonian oscillations.

We shall first of all analyze the onset and quenching of oscillations in the feedback loop in Fig. 2 and then proceed to incorporate it into a phenomenological model of the 'competing processes' of suppression and aggravation suggested by Cooper et al in connection with the response of 'essential tremor' to DBS [4].

\subsection{Genesis of Oscillations}

\section{Methods}

Invoking Liapunov's theorem of the first approximation (which is valid here as we are not dealing with a 'singular case' [17]) we explore the genesis of oscillation in the feedback loop in Fig. 2 without DBS, using small signal analysis, i.e. $y$ and $u$ exhibit small deviations from zero. In such a case, the arctan may be represented by its slope at the origin, $2 / \pi h$. The linearized loop has characteristic polynomia

$$
\begin{aligned}
& P(s)=(s+b)^{2}-\frac{2 k}{\pi h} \cdot s= \\
& =s^{2}+2\left[b-\frac{k}{\pi h}\right] \cdot s+b^{2}
\end{aligned}
$$

The root locus of $P(s)$ is shown on Fig. 3 , for $\frac{k}{\pi h}$ increasing in the range $0<\frac{k}{\pi h}<\infty$. We suggest that $h$ may be regarded as in 1:1 relation with dopamine in the basal ganglia. This has the following interpretations: 
1. For $\frac{k}{\pi h}<b$, i.e.

$$
\frac{\pi b h}{k}>1
$$

the loop is asymptotically stable, exhibiting no oscillation.

2. As $\frac{\pi b h}{k}$ passes through the critical valu

$\frac{\pi b h}{k}=1$

two complex conjugate eigenvalues migrate into the right half plane, through the values

$$
s= \pm j b
$$

\section{For}

$$
\frac{\pi b h}{k}<1
$$

the linearized loop has a pair of complex conjugate eigenvalues in the right half plane. These indicate a sinusoidal oscillation multiplied by an amplitude increasing exponentially with time. As we shall show below by describing function analysis, the saturation inherent in the arctan function limits the amplitude, so that a stable, almost sinusoidal oscillation of angular frequency

$\omega=b$

ensues.

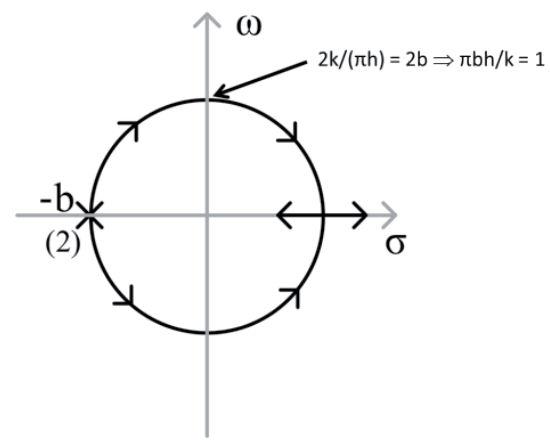

Fig. 3: Root locus corresponding to (1).

\subsection{The Describing Function and its Application}

In using the Describing Function (DF), we assume that the oscillation in the output $y$ of the nonlinear feedback loop in Fig. 2 (without DBS) may be ap- proximated to a high degree of accuracy by the expression

$y=Y_{m} \sin (\omega t)$

Conditions for this to be so have been established by Bergen and Franks [18]. They are satisfied comfortably here. Since the arctan

$$
u=\frac{2}{\pi} \arctan \left(\frac{y}{h}\right)
$$

is an odd function of $y$, the signal $u$ is an odd function of time. This means that its Fourier series consists only of sine terms, at angular frequency $\omega$ and its harmonics. In fact, due to the symmetry properties of the sine and $\arctan , u(t)$ is an inverse-repeat function of $t$ i.e.

$u\left(t+\frac{\pi}{\omega}\right)=-u(t)$

and thus, only odd harmonics at angular frequencies $3 \omega, 5 \omega, 7 \omega$ etc are generated along with the fundamental [10]. These are effectively filtered out by the $\mathrm{G}(s)$ block.

The fundamental component of $\mathrm{u}(t)$ is

$u_{f}(t)=U_{m} \sin (\omega t)$

where, by standard Fourier analysis with symmetries applied,

$U_{m}=\frac{4}{\pi^{2}} \int_{0}^{\pi} \arctan \left(\frac{Y_{m} \sin \theta}{h}\right) \cdot \sin \theta \cdot d \theta$

The DF is defined as the effective gain at fundamental frequency

$D F\left(Y_{m}\right)=\frac{U_{m}}{Y_{m}}$

The result here is

$D F\left(Y_{m}\right)=\frac{4 h}{\pi Y_{m}^{2}}\left\{\sqrt{1+\left(\frac{Y_{m}}{h}\right)^{2}}-1\right\}$

It follows from the definition of the DF as an effective gain that

$\lim _{Y_{m} \rightarrow 0} D F\left(Y_{m}\right)=\lim _{y \rightarrow 0} \frac{d u}{d y}=\frac{2}{\pi h}$

and this is readily confirmed in (13). 
The steady-state gain of the $\mathrm{G}(s)$ block for a sinusoid of angular frequency $\omega$ is $\mathrm{G}(j \omega)$. Thus, since the input to the nonlinearity and the output of the $\mathrm{G}(s)$ block are the same signal, $y$, the effective gain of the forward path under almost sinusoidal oscillation must be unity, i.e. $D F\left(Y_{m}\right) \cdot G(j \omega)=1$ or

$$
G(j \omega)=\frac{1}{D F\left(Y_{m}\right)}
$$

Equation (15) is illustrated on Fig. 4.

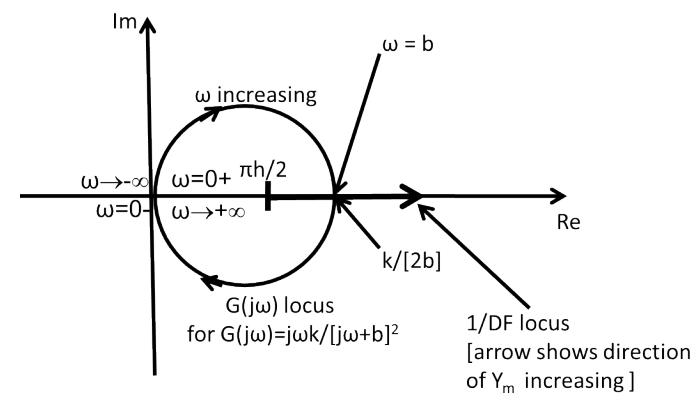

Fig. 4: Diagram to illustrate the condition shown in (15).

Provided that $\frac{\pi h}{2}<\frac{k}{2 b}$, i.e. $\frac{\pi b h}{k}<1$ (see (5)), the $\mathrm{G}(j \omega)$ and $\frac{1}{D F\left(Y_{\omega}\right)}$ loci intersect on the positive real axis for

$\omega=b$

repeating (6), and

$Y_{m}=\frac{2 k}{\pi b} \sqrt{1-\frac{\pi b h}{k}}$

For example, taking $b=k, h=0.314$ (i.e. $\frac{\pi b h}{k}=0,9865$ ), (17) yields $Y_{m}=0.0741$. The accuracy of this is shown in Fig. 5.

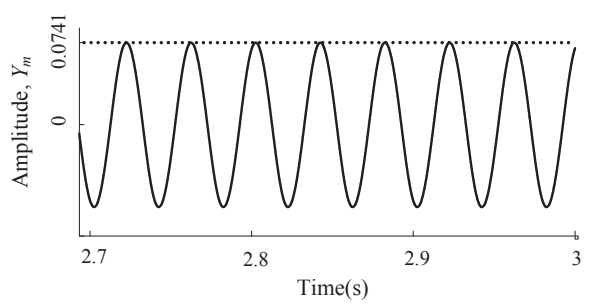

Fig. 5: Comparison of simulated model oscillations with theoretical prediction from (17) taking $b=k$ and $h=0.314$.

An informal, but yet very often accurate application of the Nyquist Criterion to Fig. 4 indicates that for

$$
\frac{\pi h}{2}<\frac{1}{D F\left(Y_{m}\right)}<\frac{k}{2 b}
$$

the 'critical point' $\frac{1}{D F\left(Y_{m}\right)}$ is encircled twice clockwise by the $\mathrm{G}(j \omega)$ locus and its conjugate, so that the closed loop is acting like a linear system having two eigenvalues in the right half plane. Thus, $\mathrm{Y}_{m}$ is increasing with time. On the other hand, for

$\frac{k}{2 b}<\frac{1}{D F\left(Y_{m}\right)}<\infty$

the closed loop is acting like a linear system having both eigenvalues in the left half plane, and so $\mathrm{Y}_{m}$ is decreasing with time. These considerations show that the almost sinusoidal oscillation with amplitude $\mathrm{Y}_{m}$ given by (17) and angular frequency $\omega=b$, is a stable limit cycle.

\subsection{Quenching the Oscillation}

We now consider the DBS waveform to be applied additively to $y$ at the input to the nonlinearity, as shown in Fig. 2. Simpson and Power [11] have shown that, provided the DBS frequency, $1 / T$, lies well above the highest significant frequency in the spectrum of $y(t)$, the effective value of $u$ is its mean value $\hat{u}$ over a DBS cycle. The original nonlinearity and the DBS source may be replaced by the Equivalent Nonlinearity, $\hat{u}$ vs $y$. Simpson and Power have developed a convolution type integral for calculating the equivalent nonlinearity, given any single-valued original nonlinear function and the DBS waveform [11]. With the simple DBS waveform employed here, however, which spends a fraction $\alpha$ of its cycle at the value $a$, a fraction $\alpha$ at the value $-a$ and a fraction $(1-2 \alpha)$ at the value 0 , it is readily appreciated that the equivalent nonlinearity is given by

$$
\begin{aligned}
& \hat{u}=\left(\frac{2}{\pi}\right)\left(\alpha \cdot \arctan \left(\frac{y+a}{h}\right)+\right. \\
& \left.+\alpha \cdot \arctan \left(\frac{y-a}{h}\right)+(1-2 \alpha) \cdot \arctan \left(\frac{y}{h}\right)\right)
\end{aligned}
$$

The DF corresponding to (20) has been evaluated by us as

$$
\begin{aligned}
& D F\left(Y_{m}\right)=\frac{4 h}{\pi Y_{m}^{2}}\left(\sqrt{\frac{Y_{m}^{2}}{h^{2}}}-1\right)- \\
& -\frac{8 \alpha h}{\pi Y_{m}^{2}}\left(\sqrt{\frac{Y_{m}^{2}}{h^{2}}+1}-\frac{Y_{m}}{h} \cdot f\right)
\end{aligned}
$$


where

$f=\sqrt{\frac{\left(c^{2}+1-b^{2}\right)+\sqrt{\left(c^{2}+1-b^{2}\right)+4 b^{2} c^{2}}}{2}}$

with

$b=\frac{a}{Y_{m}}$

and

$c=\frac{h}{Y_{m}}$

Despite its complicated appearance, the graph of $D F\left(Y_{m}\right)$ vs $Y_{m}$ decreases smoothly and monotonically as $Y_{m}$ increases for all the parameter values encountered in this study (not necessarily for all parameter values). We have summarized in Fig. 6 the conditions for oscillation and nonoscillation in terms of the relationship of $D F(0)$ to $\frac{1}{G(j b)}=\frac{2 b}{k}$. Fig. 6 shows that the oscillation is quenched provided the DBS parameters and can realize the inequality

$\frac{2}{\pi h}\left[1-\frac{2 \alpha a^{2}}{a^{2}+h^{2}}\right]<\frac{2 b}{k}$

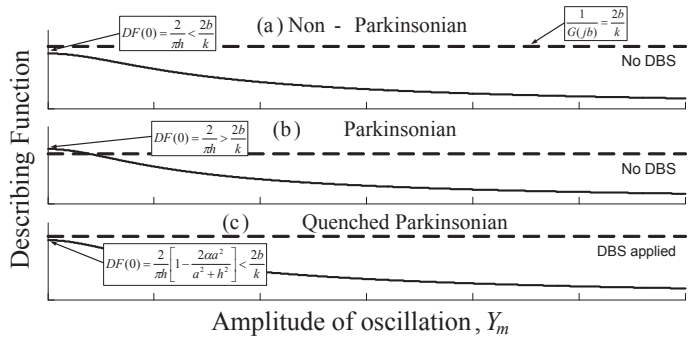

Fig. 6: Illustrating the genesis and quenching of Parkinsonian oscillations.

Given $\alpha$, the critical amplitude $a_{c}$ necessary for quenching is found by applying equality in (25):

$a_{c}=h \sqrt{\frac{\left(1-\frac{\pi b h}{k}\right)}{2 \alpha-\left(1-\frac{\pi b h}{k}\right)}}$

Since the condition for oscillation is $\frac{\pi b h}{k}<1$ (see
(5) and (17)), (26) yields a real, positive value of $a_{c}$ only if $\alpha$ exceeds the critical value

$\alpha_{c}=\frac{1-\frac{\pi b h}{k}}{2}$

Equation (27) and a scaled version of (26)

$a_{c v}=g \cdot a_{c}$

are illustrated in Fig. 7, where the scaling factor $g$ converts our dimensionless $a_{c}$ to milliamps, as used in [19]. In fitting our curve to the experimental results, we have chosen $b=k$ and calculated the values $h=0.3168$ and $g=12.492$ by minimizing a sum of squared errors criterion.

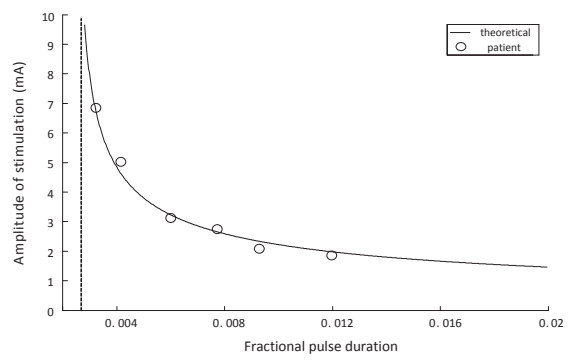

Fig. 7: Theory fitted to experimental results from [19] by minimizing a sum of squared errors criterion.
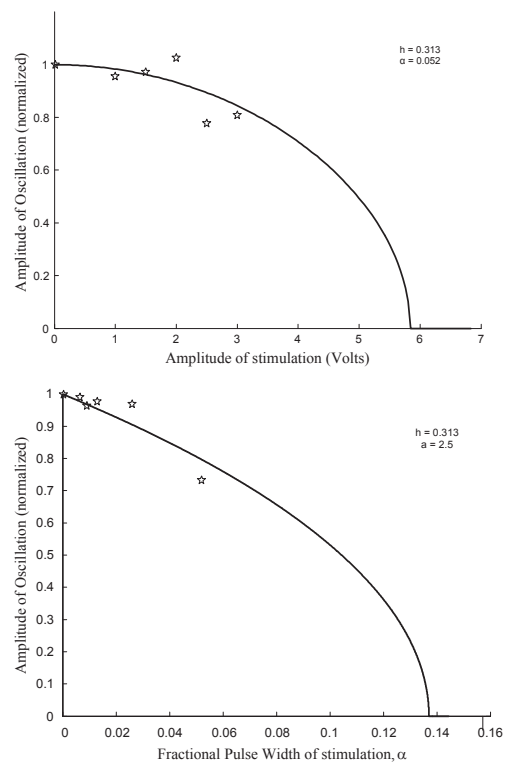

Fig. 8: a\&b compares our theory with normalized results supplied by Professor Peter Brown from the University of Oxford. 


\subsection{Response of essential tremor to DBS}

Cooper et al. have reported a very intriguing study of the response of "essential tremor" to DBS [4]. They found that the amplitude of tremor decreased at first, but then increased again as DBS voltage increased. They suggested that this could be due to "competing processes" of tremor suppression and tremor aggravation. We present here our first attempt to model this. The suppression mechanism is taken to be the same as for Parkinson's disease, discussed above. The aggravation mechanism is modeled by the nonlinear feedback loop shown in Fig. 9. As before, each linear dynamical block has transfer function $G(s)=\frac{k s}{(s+b)^{2}}$. The element subjected to DBS additively at its input is taken to be

$u=x+g x^{3}$

for which, paralleling (20), the equivalent nonlinearity is

$$
u=\left[1+6 \alpha g a^{2}\right] \cdot x+g x^{3}
$$

Corresponding to $x=X_{m} \sin (\omega t)$, the describing function is

$$
D F\left(X_{m}\right)=\left[1+6 \alpha g a^{2}\right]+\frac{3}{4} g \cdot X_{m}^{2}
$$

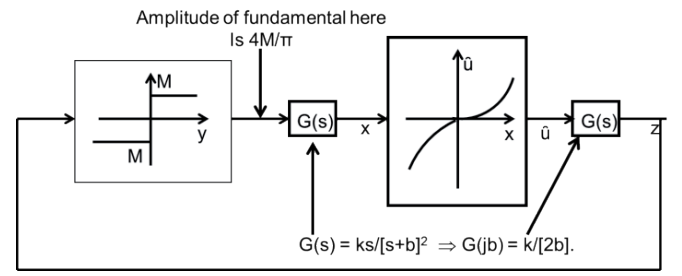

Fig. 9: Nonlinear feedback loop used to model the tremor aggravation mechanism discovered by Cooper et al. [4]

The increase as $a_{c}$ is the key to this application. As before, it can be shown that the "aggravation" loop supports an almost sinusoidal oscillation of angular frequency $\omega=b$. The amplitude of the fundamental at the output of the bang bang element is $\frac{4 M}{\pi}$ and $G(j b)=\frac{k}{2 b}$. Thus

$X_{m}=\frac{2 M k}{\pi b}$

$D F\left(X_{m}\right) . X_{m}$ gives the amplitude of the fundamental component of $\hat{u}$. Multiplying this by $G(j b)$ gives

$$
Z_{m}=\left[1+6 \alpha g a^{2}\right] \frac{M}{\pi} \cdot \frac{k^{2}}{b^{2}}+3 g\left(\frac{M}{\pi}\right)^{3} \cdot \frac{k^{4}}{b^{4}}
$$

Cooper et al. found a minimum point in the curve of

$20 \log _{10}\left[\frac{\text { tremoramplitude }}{\text { tremoramplitudewithnoDBS }}\right] v s$. Amplitudeof DBS, volts $(V)$

near the value $V=1$ [4]. We propose that

tremor amplitude $=Y_{m}(a)+w Z_{m}(a)$

where: $Y_{m}$ is output amplitude of the tremor suppression loop, which we assume is identical with that for Parkinson's disease, and that $Z_{m}$ is given by (33). To achieve 1:1 scaling between volts, $V$ and our dimensionless stimulation amplitude, $a$, we assume that the suppression loop is quenched at $a=1$. Applying equality in (25) and setting $b=k$ as we did following (17), the result is

$1-2 \alpha \cdot \frac{1}{1+h^{2}}=\pi h$

This gives the equation

$h^{3}-\frac{h^{2}}{\pi}+h-\frac{(1-2 \alpha)}{\pi}=0$

Taking $\alpha=0.024$, a value used in several experiments in [4], this has a sole positive real root at

$h=0,3043$

This is a suitable value of $h$ to give Parkinsonian-like oscillations as it gives $\frac{\pi b h}{k}=0,956<1$. We propose, therefore, our counterpoint of the Cooper et al. curve:

$$
20 \log _{10}\left[\frac{Y_{m}(a)+w Z_{m}(a)}{Y_{m}(0)+Z_{m}(0)}\right] v s . a o r V
$$

where $w$ is a scaling factor. Here we have

$$
\begin{aligned}
& Y_{m}(0)=\frac{2}{\pi} \sqrt{1-\pi(0,3043)}=0,1336 \\
& Z_{m}(a)=\left[1+6 \alpha g a^{2}\right] \frac{M}{\pi} \cdot \frac{k}{b}+ \\
& +3 g\left(\frac{M}{\pi}\right)^{3} \cdot\left(\frac{k}{b}\right)^{4}
\end{aligned}
$$


Setting $b=k$ in (41) and, in addition setting $\frac{M}{k}=1$ (which, in retrospect we have found to be reasonable, at least in this first attempt to model the aggravation process), (41) simplifies to

$$
Z_{m}(0)=1+3 g
$$

The parameters $g$ and $w$ have to be determined. For $a=0$, the left hand side of (39) is $20 \log _{10}(1)=0$, as in the graph given by Cooper et al. For $a=1$, we have

$$
\begin{aligned}
& 20 \log _{10}\left[\frac{w[1+6 \alpha g+3 g]}{0,1336+w[1+3 g]}\right]= \\
& =20 \log _{10}\left[\frac{w[1+3,144 g]}{0,1336+w[1+3 g]}\right]=-3,5
\end{aligned}
$$

The figure of -3,5dB comes from Fig. 1 in [4]. Taking the antilog, (43) gives the first equation in $w$ and $w g$ :

$$
0,0893=0,3317 w+1,139 w g
$$

Matching the $a=4$ point from the results in [4] gives which leads to the second equation in $w$ and $w g$ :

$$
20 \log _{10}\left[\frac{w[1+5,304 g]}{0,1336+w[1+3 g]}\right]=-1
$$

The solutions for $w$ and $g$ are

$$
0,119=0,1088 w+2,6302 w g
$$

The resulting curve corresponding to (39) is

$$
w=0,1325 \text { and } g=0,3001
$$

shown in Fig. 10. Some points from Cooper et al. are superimposed

\section{Conclusion}

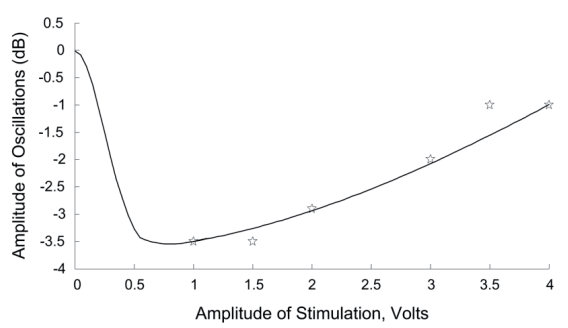

Fig. 10: Illustration of the competing processes model with six points estimated from Fig. 1 from [4].

By using well-established techniques from classical control theory, we have managed to give a reasonably accurate account of the phenomena associated with the evolution and quenching of pathological neural oscillations in the brain, associated with Parkinson's disease and Essential Tremor. There is little doubt that parallel treatments could be given for other conditions. The clinical implications of our work have yet to be established. One interesting finding, matching with very good accuracy our conversion of results due to Benabid et al [1991], is the observation that the critical DBS amplitude for quenching oscillations in the Basal Ganglia decreases monotonically with fractional pulse width, but with a "law of diminishing returns." A simple calculation (left to the reader!), establishes that the same observation applies to the energy needed to generated the DBS signal, which would be minimized if a had its maximum possible value of 0.5 . This, if it could be tolerated clinically, would have the great benefit to the patient of maximizing the time--currently between two and three years-between the operations needed to replace the stimulator when its battery has expired. In our phenomenological model of the aggravation mechanism in essential tremor, the parameters $M$ and $\mathrm{g}$ are the principal culprits. It would be fascinating to know if these have any physiological significance and whether any benign intervention could reduce either or both of them.

\section{Acknowledge}

We are very grateful to Professor Alim-Louis Benabid of the Joseph Fourier University in Grenoble, for permission to adapt his 1991 data for use in our matching exercise shown in Fig. 6; to Professor Peter Brown of the University of Oxford for the data which we have shown on Fig. 8 and 9; and to Professor Warren Grill of Duke University, North Carolina, for his hospitality to AdeP and for a very illuminating discussion of the phenomenon of essential tremor.

\section{References}

[1] Benabid, A.L., What the future holds for deep brain stimulation. Expert Rev Med Devices, 2007. 4(6): p. 895-903.

[2] Kuhn, A.A., et al., High-frequency stimulation of the subthalamic nucleus suppresses oscillatory beta activity in patients with Parkinson's disease in parallel with improvement in motor performance. J Neurosci, 2008. 28(24): p. 6165-73.

[3] Parkinson, J., An essay on the shaking palsy1817: Printed by Whittingham and Rowland for Sherwood, Neely, and Jones.

[4] Cooper, S.E., et al., A model predicting optimal parameters for deep brain stimulation in essential tremor. J Clin Neurophysiol, 2008. 25(5): p. 265-73.

[5] Carlsson, A., A Half-Century of Neurotransmitter Research: Impact on Neurology and Psychiatry (Nobel Lecture). ChemBioChem, 2001. 2(7-8): p. 484-493. 
[6] Williams, R., Alim-Louis Benabid: stimulation and serendipity. Lancet Neurol, 2010. 9(12): p. 1152.

[7] de Paor, A.M.and Lowery, Madeleine M., Can control theory throw light on Parkinson's disease and its treatment with deep brain stimulation? Signals and Systems Conference (ISSC 2009), IET Irish, 2009: p. 1-6.

[8] Plenz, D. and S.T. Kital, A basal ganglia pacemaker formed by the subthalamic nucleus and external globus pallidus. Nature, 1999. 400(6745): p. 677-82.

[9] Elgerd, 0.I., Control systems theory1967: McGraw-Hill New York.

[10] Power, H.M. and R.J. Simpson, Introduction to dynamics and control1978: McGraw-Hill UK.

[11] Simpson, R.J.and Power, H. M., Applications of high frequency signal injection in non-linear systems. International Journal of Control, 1977. 26(6): p. 917-943.

[12] Rosenblum, M. and A. Pikovsky, Delayed feedback control of collective synchrony: an approach to suppression of pathological brain rhythms. Phys Rev EStat Nonlin Soft Matter Phys, 2004. 70 (4 Pt 1): p. 041904.

[13] David, 0. and K.J. Friston, A neural mass model for MEG/EEG: coupling and neuronal dynamics. Neuroimage, 2003. 20(3): p. 1743-55.

[14] Kuramoto, Y., Chemical Oscillations, Waves, and Turbulence1984: Dover Publications. 164

[15] Hearn, A., M. Lowery, and A.M. de Paor, Some Thoughts on Electrical Interventions for the Control of Tremor in Parkinson's Dinase. Advances in Electrical and Electronic Engineering, 2011. 7(1-2): p. 334-337.

[16] de Paor, A. and J. Ringwood, A simple soft limiter describing function for biomedical applications. IEEE Trans Biomed Eng, 2006. 53(7): p. 1233-40.

[17] Liapunov, A.M., et al., Stability of motion 1966: Academic Press New York.

[18] Bergen, A. and R. Franks, Justification of the describing function method. SIAM Journal on Control, 1971. 9(4): p. 568-589.

[19] Benabid, A.L., et al., Long-term suppression of tremor by chronic stimulation of the ventral intermediate thalamic nucleus. Lancet, 1991. 337(8738): p. 403-6. 